\title{
Top quark cross section measurements with CMS
}

\author{
Javier Fernandez ${ }^{* \dagger}$ \\ Univ. of Oviedo, Facultad de Ciencias, C/ Calvo Sotelo s/n, 33007, Oviedo, Spain \\ E-mail: Javier.Fernandez@cern.ch
}

\begin{abstract}
Measurements of the inclusive top quark pair production cross section at $7 \mathrm{TeV}$ and $8 \mathrm{TeV}$ are presented. The results were obtained using CMS data collected in 2011 and 2012. The total cross section is measured in the lepton+jets, dilepton and fully hadronic channels, including the tau-dilepton and tau+jets modes. Indirect constraints on both the top quark mass and $\alpha_{s}$ are obtained through their relation to the inclusive cross section. First measurements of top quark pair production with additional b-quarks in the final state are also presented. Differential cross sections are measured and are given as functions of various kinematic observables, including the transverse momentum and rapidity of the (anti)top quark and the top-antitop system and the jets and leptons of the event final state. Multiplicity and kinematic distributions of the jets produced in addition to the top pair are also investigated. The results are combined and confronted with precise theory calculations.
\end{abstract}

XXII. International Workshop on Deep-Inelastic Scattering and Related Subjects, 28 April - 2 May 2014

Warsaw, Poland

\footnotetext{
* Speaker.

On behalf of the CMS Collaboration
} 


\section{Introduction}

A precise measurement of $t \bar{t}$ cross sections in proton-proton (pp) collisions at the Large Hadron Collider (LHC) offers a benchmark for the production mechanism of processes dominated by gluon-gluon fusion. Moreover, it can be used to validate calculations in quantum chromodynamics or to provide constraints on standard model parameters and parton distribution functions. An accurate understanding of the $t \bar{t}$ production and decay is also crucial for searches for new physics processes that show a similar event topology. The inclusive and differential cross section measurements presented in this note use pp collision data collected with the CMS detector [1] in 2011 and 2012 at a centre-of-mass energy of $7 \mathrm{TeV}$ and $8 \mathrm{TeV}$ and integrated luminosities of up to $5 \mathrm{fb}^{-1}$ and $20 \mathrm{fb}^{-1}$ respectively.

\section{Inclusive Cross Section Measurements}

With full NNLO+NNLL predictions available [2], precise measurements of the inclusive cross section are important tests for the underlying theory. The measurements are performed in distinct decay modes of the $t \bar{t}$ pair where the event selection is based on the event topology of the particular decay mode categorized by the decay of the $\mathrm{W}$ bosons from $t \bar{t} \rightarrow W^{+} W^{-} b \bar{b}$.

At $7 \mathrm{TeV}$, all decay channels except the $\tau^{+} \tau^{-}$have been performed $[3,4,5,6,8]$ and combined with the corresponding ATLAS measurement [9]. Figure 1 (left) shows a summary of $7 \mathrm{TeV}$ measurements and prediction.
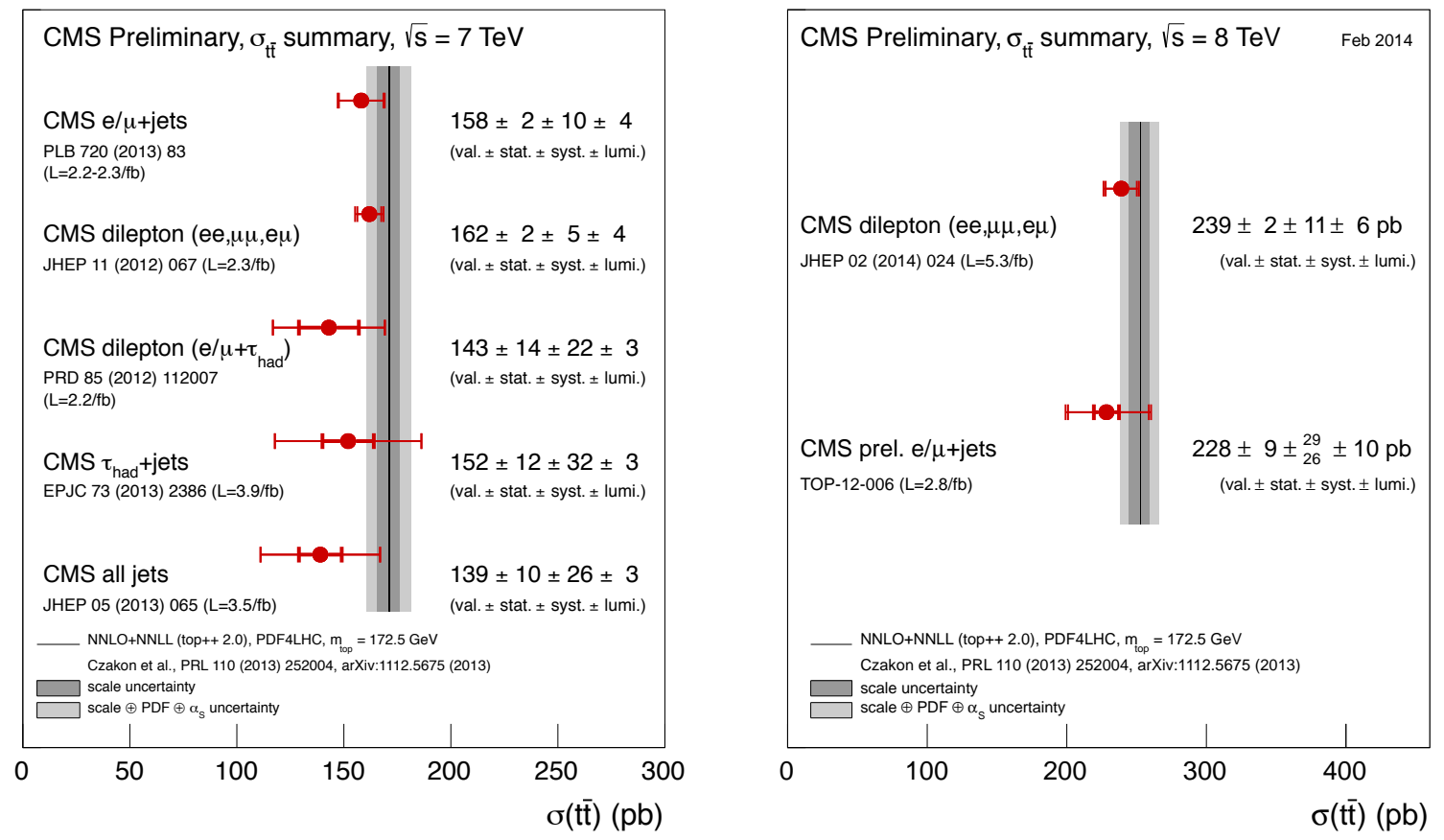

Figure 1: Summary of cross section measurements performed with the CMS detector compared to NNLO+NNLL prediction, shown by shaded bands, at $\sqrt{(s)}=7 \mathrm{TeV}$ (left) and $\sqrt{(s)}=8 \mathrm{TeV}$ (right). 
At $8 \mathrm{TeV}$, the two main channels, the so called dileptonic [11] and semileptonic [10] decay modes, have been preliminary studied. The former is characterized by both W-bosons decaying in to a lepton ( $e \pm$ or $\mu \pm$ ) and neutrino; the latter by one $\mathrm{W}$ boson decaying leptonically and one decaying hadronically (lepton + jets channel). Figure 1 (right) shows a summary of $8 \mathrm{TeV}$ predictions and measurements.

In the dileptonic decay mode, events are selected by identifying at least two oppositely charged isolated leptons with high transverse momentum $\left(p_{T}\right)$ and at least two high- $p_{T}$ jets of which at least one is identified as b-jet. Events with a low dilepton mass are removed in order to suppress heavy-flavour resonance decays. Contributions from $Z^{0}$ production are reduced by excluding the $Z^{0}$ dilepton mass window and requiring a high amount of missing $E_{T}$ in the same flavour channels. The dominant background from Drell-Yan events and from non- $W / Z^{0}$ leptons are estimated from data, single-top-quark production and diboson background events are taken from simulation. A result of $\sigma_{t \bar{t}}=227 \pm 3$ (stat.) \pm 11 (syst. $) \pm 10$ (lumi) pb, for a top-quark mass of $172.5 \mathrm{GeV} / \mathrm{c}^{2}$ with $5 \mathrm{fb}^{-1}$ of data is obtained.

In the semileptonic channel, events that yield exactly one isolated identified lepton and at least four high- $p_{T}$ jets of which one is identified as a b-jet, are selected. The shape of the QCD multijet background is estimated from a control region with non-isolated leptons. The latter, the $t \bar{t}$ signal shape and the remaining background (from simulation) is used in a binned likelihood fit of the lepton-b-jet invariant mass to extract the $t \bar{t}$ cross section. For a top-quark mass of $172.5 \mathrm{GeV} / \mathrm{c}^{2}$ the $t \bar{t}$ cross section is measured to be $\sigma_{t \bar{t}}=229 \pm 9$ (stat. $) \pm_{26}^{29}$ (syst. $) \pm 10($ lumi $) p b$.

\section{Determination of $\alpha_{s}$ as from $t \bar{t}$ cross sections}

The strong coupling constant $\alpha_{s}$ is the only free parameter in the QCD Lagrangian besides the quark masses. The dependence of $\alpha_{s}(Q)$ on the energy scale of a process $\mathrm{Q}$ is given by the renormalization group equation. Only very few measurements allow $\alpha_{s}$ to be tested at high scales, e.g. $Q \geq m_{t}$. The production of $t \bar{t}$ pairs at high scales and its precisely measured cross section [5] can be combined with predictions at NNLO to extract as $\alpha_{s}$ [13].

The method is taking advantage of an expected sensitivity of $\sigma_{t \bar{t}}$ to $\alpha_{s}$ due to correlations with the gluon parton density function (PDF). For different PDFs, a joined likelihood fit is performed using the predicted cross section as a function of $\alpha_{s}\left(m_{Z}\right)$ and the measured $t \bar{t}$ cross section. For the first time, by constraining the pole-top mass to the latest average from direct measurements and using the PDF set NNPDF2.3, a value of $\alpha_{s}\left(m_{Z}\right)=0.1151 \pm_{0.0032}^{0.0033}$ is extracted, in agreement with the world average. The measurement in the top mass is $m_{t}^{\text {pole }}=176.6_{-3.4}^{+3.8} \mathrm{GeV} / \mathrm{c}^{2}$.

\section{Differential Cross Section Measurements}

The high $t \bar{t}$ production rate at the LHC allows for measurements of the $t \bar{t}$ production cross section as a function of various kinematic observables. These differential cross sections are important tests for QCD predictions, enhance the sensitivity to new physics. The measurements presented here at $7 \mathrm{TeV}$ [7] and $8 \mathrm{TeV}[17,16]$ are performed in the dileptonic and the semileptonic decay modes and follow a similar event selection as the inclusive cross section measurements. A kinematic reconstruction of the top-pair system is performed and cross sections are extracted using a 

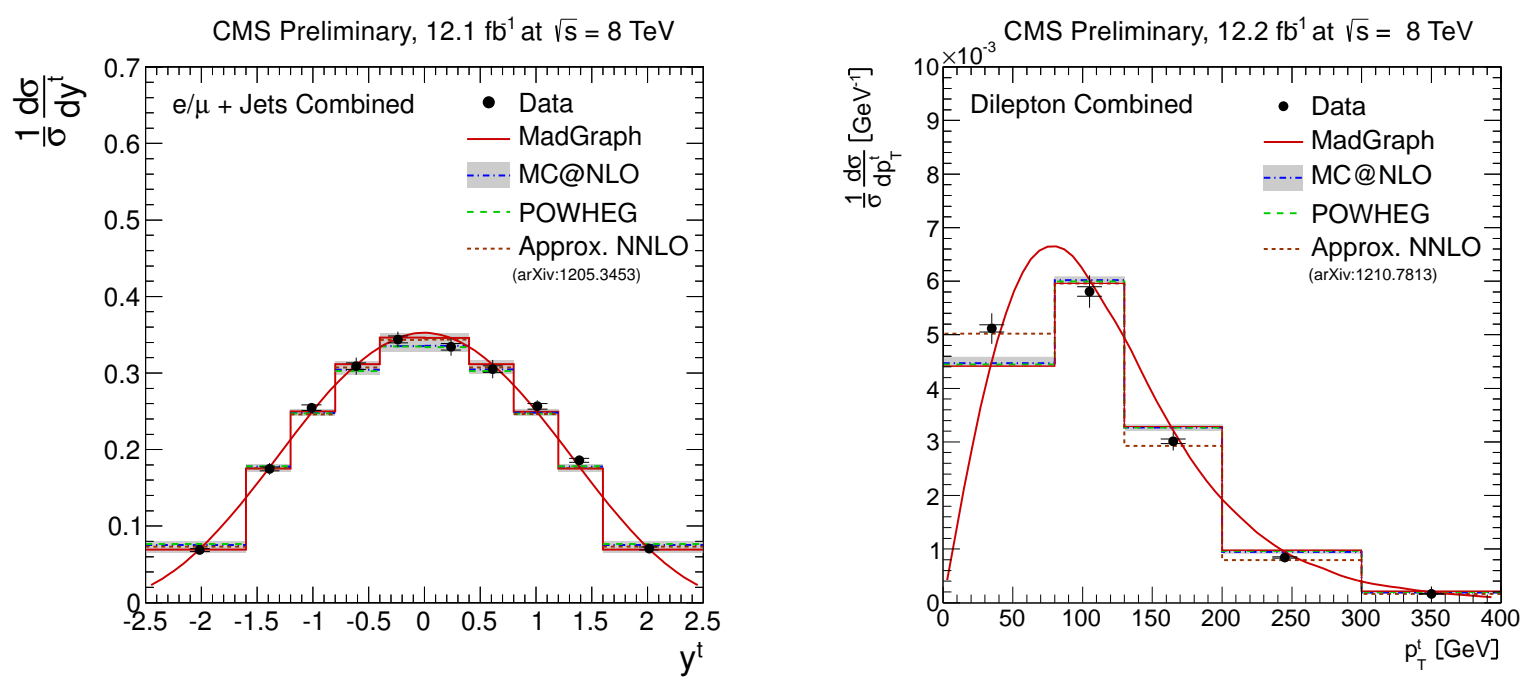

Figure 2: Normalized differential $t \bar{t}$ production cross section at $8 \mathrm{TeV}$ as a function of top rapidity $\left(y_{t}\right)$ measured in the semileptonic decay mode (left) and as a function of top transverse momentum $\left(p_{T}\right)$ measured in the dileptonic decay mode (right) compared to different predictions (MADGRAPH interfaced with Pythia, MC@NLO interfaced with Herwig, POWHEG interfaced with Pythia, and approx NNLO). Data is presented as filled symbols. Inner (outer) error bars indicate statistical (combined statistical and systematic) uncertainties.

regularized unfolding technique. The results are normalized to the in-situ measured cross section to reduce normalization uncertainties. In general, all distributions show good agreement between data and theory predictions (MADGRAPH interfaced with Pythia, MC@NLO interfaced with Herwig, POWHEG interfaced with Pythia) as shown in Figure 2. The normalized cross section as a function of top $p_{T}$ seems to favor approx. NNLO predictions in both $\sqrt{s}=7$ and $8 \mathrm{TeV}$.

\section{Jet Multiplicities in Top Pair Events}

A high fraction of events at the LHC yields additional hard jets from initial and final state radiation (ISR, FSR). The understanding of ISR and FSR is crucial not only for high energetic top or Higgs production but for all processes involving high jet multiplicities. A measurement of $t \bar{t}$ production with additional jets is performed in the semileptonic decay mode at $\sqrt{s}=7 \mathrm{TeV}$ [12] and the dileptonic decay mode at $\sqrt{s}=7$ [14] and $8 \mathrm{TeV}[18]$.

The analysis strategy follows a similar event selection as the inclusive cross section measurements. Figure 3 shows an increasing difference between several predictions (MADGRAPH interfaced with Pythia, MC@NLO interfaced with Herwig, POWHEG interfaced with Pythia) with increasing jet multiplicity. For high multiplicities MC@NLO interfaced with Herwig seems to underestimate the data in both decay modes, while the rest of generators show predictions consistent with data. 

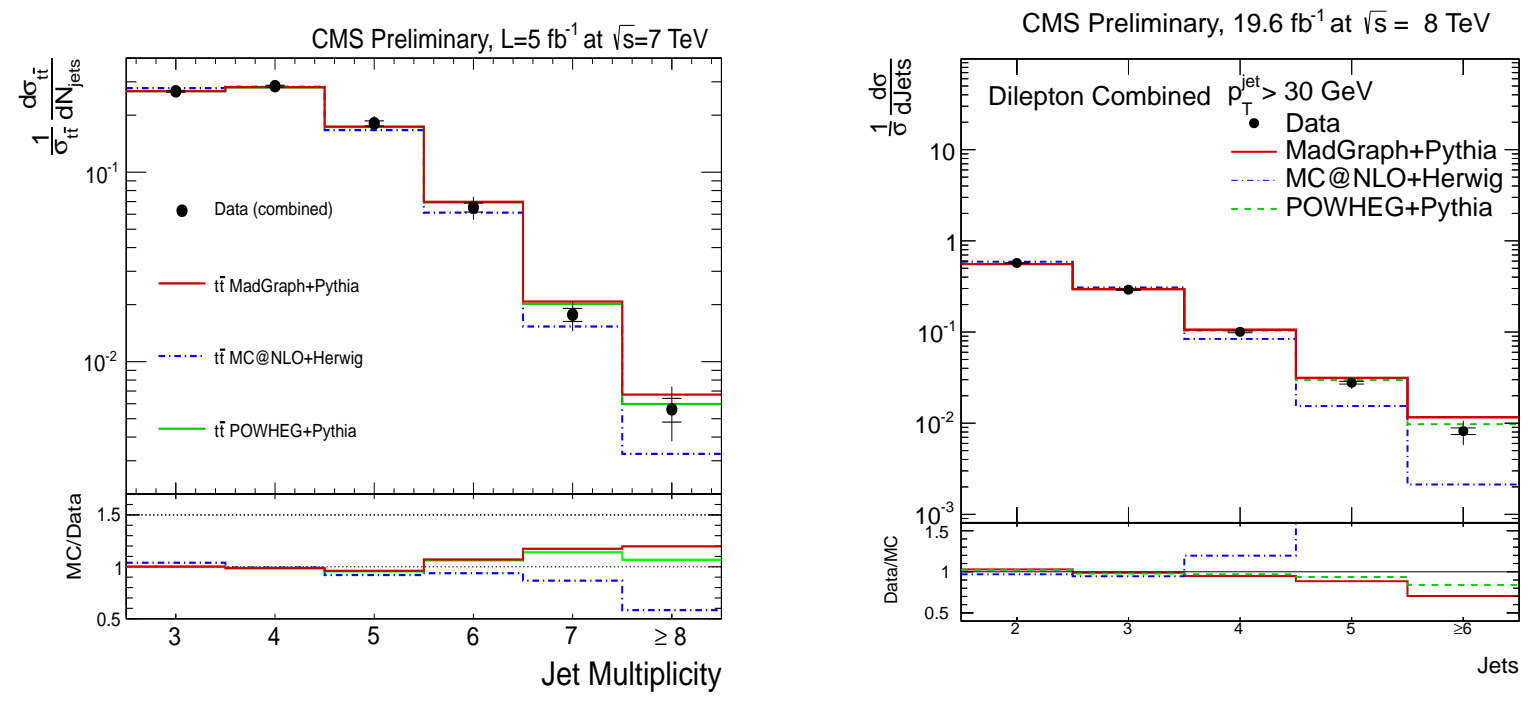

Figure 3: Normalized differential $t \bar{t}$ production cross section as a function of jet multiplicity, measured in the semileptonic decay mode with $p_{T}^{\text {jet }}>35 \mathrm{GeV} / \mathrm{c}$ at $7 \mathrm{TeV}$ (left) and the dileptonic decay mode with $p_{T}^{\text {jet }}>30 \mathrm{GeV} / \mathrm{c}$ (right) compared to different predictions (MADGRAPH interfaced with Pythia, MC@NLO interfaced with Herwig, POWHEG interfaced with Pythia). Data is presented as filled symbols. The inner (outer) error bars indicate statistical (combined statistical and systematic) uncertainties.

\section{Measurement of the Cross Section Ratio $\sigma(t \bar{t} b \bar{b}) / \sigma(t \bar{t} j j)$}

The cross section ratio (allowing a large cancellation of uncertainties) $\sigma(t \bar{t} b \bar{b}) / \sigma(t \bar{t} j j)$ has been measured by the first time in the visible phase space corresponding to the detector acceptance, and corrected to particle level. For events with at least four reconstructed jets and $2 \mathrm{~b}$-tags, the measurement is performed in the dilepton channel by means of a fit to the measured b-tagging algorithm discriminator at $8 \mathrm{TeV}$ [19] and using the b-tag multiplicity at $7 \mathrm{TeV}$ [15]. The result of the measurement at $8 \mathrm{TeV}$ is $0.023 \pm 0.003$ (stat.) \pm 0.005 (syst.) and $0.022 \pm 0.004$ (stat.) \pm 0.005 (syst.) for a minimum $p_{T}$ of 20 and $40 \mathrm{GeV} / \mathrm{c}$, respectively. Measurements of the absolute cross sections $\sigma(t \bar{t} b \bar{b})$ and $\sigma(t \bar{t} j j)$ are also performed.

\section{Summary}

A variety of cross section measurements has been performed using pp collision data collected with the CMS experiment at a centre of mass energy of 7 and $8 \mathrm{TeV}$. Inclusive top pair cross sections have been measured in nine decay channels in total and up to a high precision, in good agreement with predictions. These precise results were used to extract the strong coupling constant from the $t \bar{t}$ cross section. Differential cross sections as well as jet multiplicity distributions were measured in the dileptonic and semileptonic decay modes and provide a basis for precision test of the Standard Model and searches for new physics. Besides, a first measurement of the $\sigma(t \bar{t} b \bar{b}) / \sigma(t \bar{t} j j)$ cross section ratio has been performed, a process of great importance for the $t \bar{t} H \rightarrow t \bar{t} b \bar{b}$ search since it represents an irreducible background as well as a test of perturbative QCD theory. 


\section{References}

[1] The CMS Collaboration, The CMS experiment at the CERN LHC, JINST 03 (2008) S08004.

[2] Michal Czakon, Paul Fiedler, Alexander Mitov, The total top quark pair production cross-section at hadron colliders through $O\left(\alpha_{S}^{4}\right), P R L 110$ (2013) 252004 [hep-ph/1303 . 6254]

[3] The CMS Collaboration, Measurement of the ttbar production cross section in pp collisions at $\sqrt{(} s)$ = 7 TeV with lepton + jets final states, Phys. Lett. B 720 (2013) 83CMS PAS TOP-11-003 [hep-ex/1212.6682].

[4] The CMS Collaboration, Measurement of the top-antitop production cross section in the tau+jets channel in pp collisions at $\sqrt{(s)}=7$ TeV, Eur. Phys. J. C73 (2013) 2386[hep-ex/1301.5755].

[5] The CMS Collaboration, Measurement of the ttbar production cross section in the dilepton channel in pp collisions at $\sqrt{(s)=7 ~ T e V, J H E P} 11$ (2012) 067[hep-ex/1208.2671].

[6] The CMS Collaboration, Measurement of the top quark pair production cross section in pp collisions at $\sqrt{(s)}=7 \mathrm{TeV}$ in dilepton final states containing a tau, Phys. Rev. D 85 (2012) 112007[hep-ex/1203.6810].

[7] The CMS Collaboration, Measurement of differential top-quark pair production cross sections in pp collisions at $\sqrt{(s)}=7$ TeV, Eur. Phys. J. C73 (2013) 2339[hep-ex/1211.2220].

[8] The CMS Collaboration, Measurement of the ttbar production cross section in the all-jet final state in pp collisions at $\sqrt{s}=7$ TeV, JHEP 1305 (2013) 065[hep-ex/1302 . 0508].

[9] The CMS Collaboration, Combination of ATLAS and CMS top-quark pair cross section measurements using proton-proton collisions at $\sqrt{s}=7 \mathrm{TeV}$, CMS PAS TOP-12-003.

[10] The CMS Collaboration, Cross section measurement in the l+jets channel at $8 \mathrm{TeV}, \mathrm{CMS} P A S$ TOP-12-006.

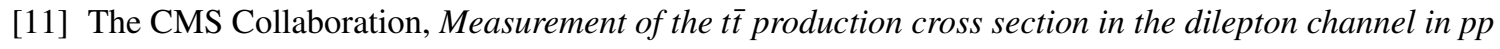
collisions at $\sqrt{s}=8 \mathrm{TeV}$, JHEP 02 (2014) 024[hep-ex/1312 . 7582].

[12] The CMS Collaboration, Measurement of jet multiplicity distributions in t t-bar production in pp collisions at sqrt(s) = 7 TeV, CMS PAS TOP-12-018 [hep-ex/1403.3171].

[13] The CMS Collaboration, Determination of the top-quark pole mass and strong coupling constant from the ttbar production cross section in pp collisions at $\sqrt{s}=7$ TeV, Phys. Lett. B 728 (2013) 496[hep-ex/1307.1907].

[14] The CMS Collaboration, Measurement of jet multiplicity in dilepton events at 7TeV, CMS PAS TOP-12-023.

[15] The CMS Collaboration, Measurement of $\sigma(t t b b) / \sigma(t t j j)$ at 7TeV, CMS PAS TOP-12-024.

[16] The CMS Collaboration, Measurement of the differential ttbar cross section in the lepton+jets channel at $8 \mathrm{TeV}, \mathrm{CMS}$ PAS TOP-12-027.

[17] The CMS Collaboration, Measurement of the differential ttbar cross section in the dilepton channel at $8 \mathrm{TeV}, \mathrm{CMS}$ PAS TOP-12-028.

[18] The CMS Collaboration, Measurement of the Jet Multiplicity in Dileptonic Top Quark Pair events at 8 TeV, CMS PAS TOP-12-041.

[19] The CMS Collaboration, Measurement of Cross Section Ratio $\sigma(t t b b) / \sigma(t t j j)$ in pp Collisions at 8 TeV, CMS PAS TOP-13-010. 